\title{
First protozoa-trapping plant found
}

Some 450 species of insectivorous plants Shave been discovered since Darwin published his work on the subject in $1875^{1}$. We report here the first known case of a plant that traps and digests protozoa.

Insectivorous plants colonize nutrientpoor places, and their major source of phosphorus, nitrogen and other elements is trapped and digested insects ${ }^{2,3}$. Extravagant trap-like structures are seen in the genus Genlisea (a member of the Lentibulariaceae, with the carnivorous genera Pinguicula and Utricularia) which occurs mainly in nutrient-poor white sands and moist rock outcrops in South America and tropical Africa. However, proof of the plant's carnivorous nature was previously lacking.

Genlisea species are rare in the wild and difficult to cultivate. Most form a small rosette about three centimetres in diameter, close to the ground, with linear or spatulate leaves. The yellow or violet flowers, similar to those of the related Antirrhinum, are borne on an inflorescence some $20 \mathrm{~cm}$ high.

When the rosette of Genlisea is dug up, pale bundles of root-like organs up to 15 $\mathrm{cm}$ long are revealed (Fig. 1a). However, the plant is rootless and these organs are subterranean leaves, highly modified and lacking chlorophyll ${ }^{4-6}$. The long, solid, basal part of the leaf attached to the rhizome opens into a hollow utricle. This contracts into a narrow tubular channel (the 'neck piece') which divides into two arms at an angle of $90-130^{\circ}$ so that the apical part of the leaf is shaped like an inverted letter ' $Y$ '. The helically contorted arms are hollow, with an average inside diameter of 200 micrometres. The arms bear slit-like openings (width $400 \mu \mathrm{m}$, height $180 \mu \mathrm{m}$ ) lined with rows of hairs pointing towards the utricle, and numerous glands.

Since Darwin's time, it has been postulated that these specialized leaves are traps for catching prey, but there has been no proof of carnivory. Arthropod remnants have only exceptionally been found in the traps. This, and the dimensions of the traps, suggested to us that the subterranean leaves might function as highly
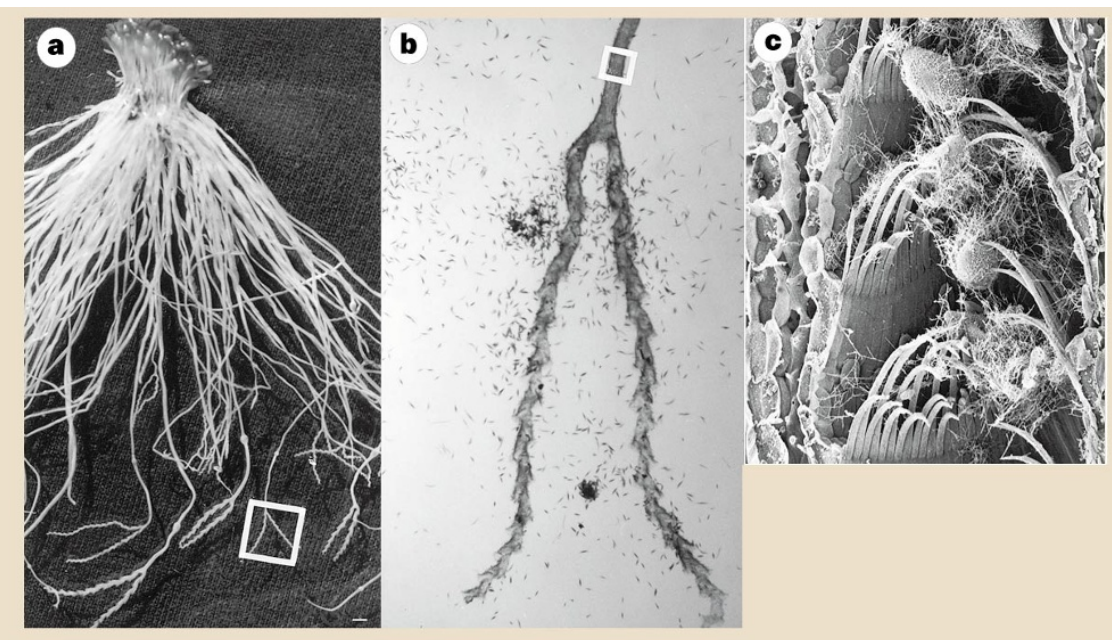

Figure 1 Genlisea aurea protozoan traps. a, Green rosette of assimilating leaves (on top) and a large bundle of root-like subterranean leaves acting as traps. b, A single Genlisea trap with protozoa attracted and concentrated around the utricle and the tube. c, Scanning electron micrograph of the tube after a 'feeding' experiment with Paramecium caudatum, fixed with osmium tetroxide. Captured protozoa are visible. The rows of long hairs prevent escape.

specialized traps for catching protozoa.

To test this idea, we cultivated species of Genlisea in a greenhouse. Laboratory experiments were conducted with G. aurea, $G$. violacea and G. margaretae. Intact plants were placed in a Petri dish and ciliates such as Blepharisma americana were added.

The root-like subterranean organs of Genlisea proved attractive to protozoa (Fig. 1b). A few minutes after starting the experiment, numerous protozoa had already entered the traps (Fig. 1c). In contrast, living roots from plants occurring in the same habitats — such as Eriocaulon plumale failed to attract ciliates.

The presence of acid phosphatases and esterases in G. africana ${ }^{7}$ suggests that the ciliates are digested. Additional experiments proved the existence of an attractant and indicated that Genlisea attracts protozoa chemotactically, trapping them in its subterranean leaves.

Genlisea can therefore be regarded as a highly specialized protozoan trap. Experiments using ciliates marked with the isotope sulphur-35 demonstrated its uptake by the trap leaves of Genlisea. After two days, ${ }^{35} \mathrm{~S}$ was traceable in the rosette leaves.

Field investigations at a natural site in the northern part of the Ivory Coast in west Africa indicate that, at this site at least, nine different ciliate species, as well as other protozoa, are trapped in large amounts by $G$. stapfii. Thus, 125 years after Charles Darwin's initial postulations, the puzzle of Genlisea's feeding habits seems to have been solved.

Wilhelm Barthlott, Stefan Porembski, Eberhard Fischer, Björn Gemmel Botanisches Institut der Universität Bonn,

Meckenheimer Allee 170, D-53115 Bonn, Germany e-mail:barthlott@uni-bonn.de

2. Juniper, B. E., Robins, R. J. \& Joel, D. M. The Carnivorous Plants (Academic, London, 1989).

3. Lloyd, F. E. The Carnivorous Plants (Chronica Botanica, Waltham, 1942). Jena, 1928).

5. Warming, E. Medd. Kjøb. 1, 33-70 (1874)

6. Reut, M. S. Bot. Helv. 103, 101-111 (1993).

7. Heslop-Harrison, Y. in Lysosomes in Biology and Pathology 4 (eds Dingle, J. T. \& Dean, R. T.) 525-578 (North Holland, Amsterdam, 1975).
1. Darwin, C. Insectivorous Plants (Murray, London, 1875).

4. Goebel, K. Organographie der Pflanzen part 1, 3rd edn (Fischer,

\section{Domains of rasGAP and rhoGAP are related}

Guanine-nucleotide-binding $(\mathrm{G})$ proteins are 'switched off' by the hydrolysis of their bound GTP. The GTP bound to the G protein Ras is hydrolysed intrinsically at a very slow rate ${ }^{1}$, so, in vivo, Ras is turned off by GTPase-activating proteins (GAPs). The structures of GTPase-activating domains (GAP domains), which usually occur as parts of larger proteins, indicate that the Ras and Rho families of small $G$ proteins and their GAPs evolved in parallel.

Determination of the structure of the GAP domain from p120GAP, first in isolation $^{2}$ and then in a Ras-rasGAP complex ${ }^{3}$, showed that an arginine residue from the GAP domain plays a crucial role in cataly$\operatorname{sis}^{1,3}$. GAPs for the Rho family have also been characterized and the structure of a GAP domain from p50rhoGAP has been determined in isolation ${ }^{4}$ and in a transition-state complex with RhoA $A^{5}$ The rhoGAP domain provides a crucial arginine residue to the active site of RhoA.

A domain homologous to rhoGAPs but lacking GAP activity is found in the regulatory p85 subunit of phosphatidylinositol-3-OH kinase. The structure of this rhoGAP-like domain from p $85 \alpha$, called 


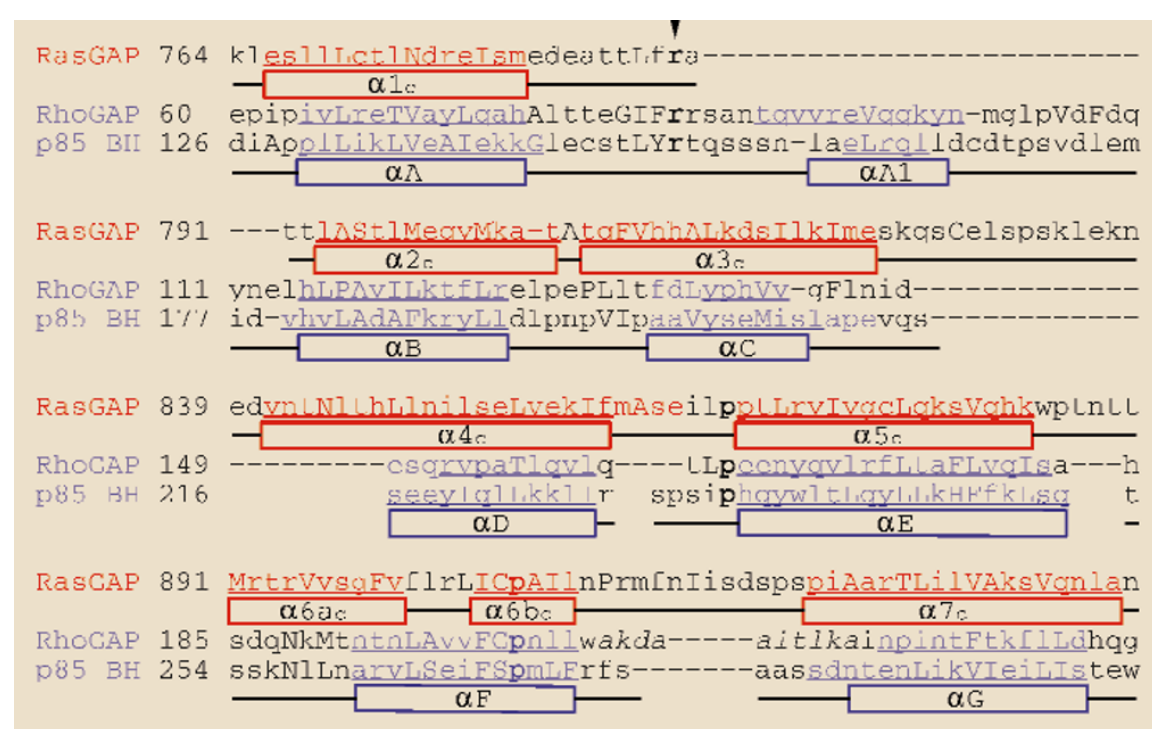

Figure $1 \mathrm{~A}$ structurally based sequence alignment of comparable regions of the rasGAP domain from p120GAP (PDB code: 1wer), the rhoGAP domain of p50rhoGAP (PDB code: 1rgp) and the BH rhoGAP-like domain of the p85 subunit of phosphatidylinositol-3-OH kinase (PDB code: 1pbw). The computer program COMPARER ${ }^{78}$ provided the alignment. $\alpha$ Helices are underlined and shown in red (rasGAP) or blue (rhoGAP, p85 BH); $3_{10}$ helices are not underlined. The nomenclature for helices is as originally defined ${ }^{2,6,4}$. Residues labelled in upper-case lettering are inaccessible to solvent. The position of Arg 789 (rasGAP)/Arg 85 (rhoGAP)/Arg 151 (p85 BH) is indicated by the arrow. Residues between helix $\alpha F$ and $\alpha G$ are disordered in the rhoGAP structure (in italics).

p85 $\mathrm{BH}$ (where $\mathrm{BH}$ indicates a BCRhomology domain), has also been determined $^{6}$. The release of the coordinates for the rhoGAP and rasGAP domains allowed us to compare their structures. We found that the rhoGAP and rasGAP domains are clearly related (my Fig. 1 and Fig. 1 in the letter below by Rittinger et al.) and must have derived from the same ancestral protein.

To see the equivalence of the structures more clearly (in Fig. 1 of Rittinger et al.), place your finger on the top of helix $\mathrm{F}$ (of rhoGAP) and imagine pushing it away from you and down so that it lies more nearly parallel to helix $G$ and between helices $G$ and $\mathrm{E}$ (helices $\mathrm{E}$ and $\mathrm{A}$ then move to the left). The rasGAP and rhoGAP sequences (in my Fig. 1) are only 6\% identical, whereas rasGAP and $\mathrm{p} 85 \mathrm{BH}$ are $13 \%$ identical (the two rhoGAP-like domains, whose similarity was identified at the sequence level, have a $17 \%$ sequence identity).

In the transition-state-analogue complexes of both Ras-rasGAP ${ }^{3}$ and Rho-rhoGAP ${ }^{5}$, helix $\alpha 6 / \alpha \mathrm{F}$ interacts with the switch II loop on Ras/Rho. This interaction helps to stabilize the conformation of the switch II loop which provides residue Gln 61 ( $G \ln 63$ in Rho) for the active site. The large structural shift in the relative orientation of helix $\alpha 6 / \alpha \mathrm{F}$ of rasGAP/rhoGAP may have occurred to compensate for the different structures of the switch II loop in Ras and Rho.

This equivalence in structure points to a parallel evolution by Ras and Rho G proteins and their GAPs.

\section{Benjamin Bax}

Department of Crystallography, Birkbeck College,

Malet Street, London WC1E 7HX, UK

e-mail:b.bax@mail.cryst.bbk.ac.uk

\footnotetext{
. Ahmadian, M. R., Stege, P., Scheffzek, K. \& Wittinghofer, A. Nature Struct. Biol. 4, 686-689 (1997).

2. Scheffzek, K., Lautwein, A., Kabsch, W., Ahmadian, M. R. \& Wittinghofer, A. Nature 384, 591-596 (1996)

3. Scheffzek, K. et al. Science 277, 333-338. (1997)

4. Barrett, T. et al. Nature 385, 458-461 (1997).

5. Rittinger, K., Walker, P. A., Eccleston, J. F., Smerdon, S. J. \& Gamblin, S. J. Nature 389, 758-762 (1997).

6. Mussachio A., Cantley, L. C. \& Harrison, S. C. Proc. Natl Acad. Sci. USA 93, 14373-14378 (1996).

. Sali, A., \& Blundell, T. L. J. Mol. Biol. 212, 403-428 (1990)

8. Zhu, Z.-Y., Sali,A. \& Blundell, T. L. Protein Engng 5, 43-51 (1992).
}

The Ras superfamily of monomeric GTPase proteins comprises six subfamilies, each with specific cellular functions, but all these subfamilies share a common fold and a common mechanism for hydrolysing GTP. Unlike their cognate guanine-nucleotidebinding (G) proteins, GTPase-activating proteins (GAPs) specific for the Rho or Ras subfamilies show no significant homology at the level of protein sequence. However, we have now noticed a structural similarity which suggests that, like their $G$ proteins, these GAPs have evolved from a common ancestor.

Small G proteins belonging to the Ras superfamily act as signalling molecules con-

\section{Support for shared ancestry of GAPs}

trolling a wide range of biological processes. Crucial to their signalling function is the conformational switching that takes place between their active, GTP-bound and inactive, GDP-bound forms ${ }^{1}$. They have a slow, intrinsic rate of GTP hydrolysis which is substantially accelerated by GAPs specific to each subfamily. Crystallographic coordinates for rhoGAP and rasGAP are now available ${ }^{2,3}$ and we have compared them using the program LSQMAN (ref. 4).

Superposition of the two GAPs shows that they share a core structure made up of seven $\alpha$-helices (Fig. 1). The $\alpha$-helices making up this core pack against each other in a related, but not identical, fashion. However, the automated alignment puts the catalytic arginine residues (R85 in rhoGAP and R789 in rasGAP) into approximately the same position and, by implication, locates the active sites of the bound $\mathrm{G}$ proteins.

It is particularly interesting to note that in both structures the catalytic arginine residue is located on a surface loop and is preceded by two bulky, hydrophobic residues (isoleucine and phenylalanine at residues 83 and 84 , respectively, in rhoGAP, and leucine and phenylalanine at positions 787 and 788, respectively, in rasGAP). These residues are held in a hydrophobic clamp made up of residues from the $\mathrm{B}(2 \mathrm{c}), \mathrm{E}(5 \mathrm{c})$ and $\mathrm{F}(6 \mathrm{c})$ helices that act to anchor the catalytic arginine loop to the core of the domain.

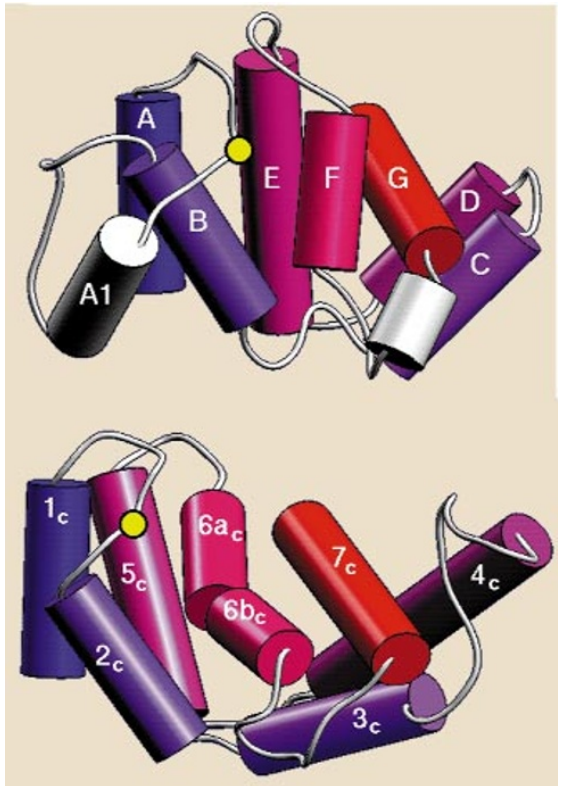

Figure 1 The common core structures of the catalytic domains of p50rhoGAP (top) and p120rasGAP (bottom), as aligned by the computer program LSQMAN, in helical-tube representation. Helical segments forming the core structure (helices $A-G$ and $1 \mathrm{c}$ to $7 \mathrm{c}$ in rhoGAP and rasGAP, respectively) are coloured from blue at their amino termini to red at their carboxy termini. Helical insertions that do not form part of the core motif are grey. The positions of the catalytic arginines R85(rhoGAP) and R789(rasGAP) are indicated by yellow circles. 